\title{
WORK SITUATION, WORK ABILITY AND EXPECTATION OF RETURNING TO WORK IN PATIENTS WITH SYSTEMIC AUTOIMMUNE MYOPATHIES
}

\author{
Rafael Alves Cordeiro ${ }^{1, \star}$, Frida Marina Fischer ${ }^{1}$, Samuel Katsuyuki Shinjo ${ }^{1}$ \\ 1.Universidade de São Paulo, São Paulo (SP), Brazil. \\ *Corresponding author: rafael19abc@hotmail.com
}

\section{BACKGROUND}

The effects of systemic autoimmune myopathies (SAMs) on work-related outcomes have not been extensively studied to date. Herein, we aimed to document the work situation, the work ability and the expectation of returning to work among patients with SAMs and to identify the factors associated with each of these main outcomes.

\section{METHODS}

This is a single-center, cross-sectional study that included adult patients with SAMs (EULAR/ACR-2017 classification criteria), enrolled from September 2019 to March 2020. The work situation was ascertained via a structured questionnaire. For those who were working, we applied the work ability index (WAI); and for those who were unemployed, in disease leave or retired, we applied the return-to-work self-efficacy questionnaire (RTW-SE). The following parameters were used to assess disease activity: physician global activity (Likert scale), muscle strength (MMT-8), functionality (HAQ-DI), creatine phosphokinase levels, and MYOACT visual analogue scales. We also collected data on: sociodemographic variables, myositis subgroup, disease duration, prednisone dose, body mass index, fatigue (FSS), mental health (DASS-21), quality of life (WHOQOL-BREF), absenteeism and presenteeism (WPAI), and comorbidities. Logistic regression and multiple linear regression models were created and the analyzes were performed at a significance level of 0.05 .

\section{RESULTS}

The study sample consisted of 75 patients with SAMs (female: $66.7 \%$; age: $44.1 \pm 9.4$; disease duration: $6.2 \pm 4.5$ years). Of these, 33 (44\%) patients were working, while 42 (56\%) were out of work (unemployed: 62\%; temporary sickness benefit recipients: 24\%; retired: $14 \%$ ). In the logistic regression model, one point increase in the HAQ-DI, decreases the chance of working by $66 \%$ $(\mathrm{OR}=0.34,95 \% \mathrm{Cl}=0.16-0.74, \mathrm{p}=0.007)$. Among those who were working $(\mathrm{n}=33)$, the mean WAI score was $33.5 \pm 6.9$. In the linear regression model, the presence of fatigue, fibromyalgia and diabetes lead to an average decrease of 3.22, 4.72 and 3.90 points in the WAI, respectively $(p<0.01)$. For those not working $(n=42)$, the mean RTW-SE score was $42.8 \pm 12.4$. More than 12 years of education and the presence of cutaneous manifestation are associated with an average increase of 10.9 and 10.57 points, respectively, in the expectation of returning to work. Moreover, one point increase in HAQ decreases the expectation by 4.69 points ( $p<0.05$ ).

\section{CONCLUSION}

To our knowledge, this is the most comprehensive study which addressed work-related outcomes in SAMs. Our findings highlight the poor work participation in a well-characterized sample of patients with SAMs and the urgent need to formulate strategies to mitigate the burden of this disease in a developing country.

\section{KEYWORDS}

Dermatomyositis, Polymyositis, Employment, Work capacity evaluation, Return to work. 